\title{
La prospectiva como diseño de lo intangible. El caso de CENTRO
}

\author{
Paniagua, Karla
}

PhD candidate and research coordinator - Centro de Diseño, Cine y Televisión, kpaniagua@centro.edu.mx

\begin{abstract}
Resumen
Se reseña la experiencia de investigación y confección de la especialidad en Diseño del mañana, programa de posgrado destinado a la formación de expertos en solución de problemas sociales con base en los métodos de la prospectiva, conjunto de herramientas y técnicas destinadas a sistematizar la imaginación para el diseño de futuros posibles, probables o preferibles.. Se concluye que en sí misma, la experiencia aspira a constituir una solución a un problema cultural signiticativo que permea todos los ámbitos de la vida: el de la visión a corto plazo. La presentación abarca los antecedentes del proyecto, la descripción del proceso de investigación, la exposición del programa y el estado actual del proyecto, que ocurre en el marco institucional de CENTRO, institución dedicada a la profesionalización de la Economía creativa.
\end{abstract}

Entre los resultados más relevantes de esta experiencia pueden señalarse, además del programa en sí, que ya se encuentra vigente y debidamente validado por la Secretaría de Educación Pública en México, la conformación de un claustro de profesores nacionales e internacionales altamente motivados y comprometidos por la experiencia; la integración de un consejo consultivo con representación de los más importantes think-tanks de futurología alrededor del mundo (la World Future Society, la World Future Studies Federation, Institute for the Future, entre otros) y la incorporación de CENTRO a la discusión internacional en materia de futuros, entendiendo los futuros como objetos de diseño complejos que pese a no existir, son integrados-conjurados-a la realidad material mediante narrativas, objetos, prototipos, escenarios y otras expresiones materiales que contribuyen a guiar la toma de decisiones en el presente.

El diseño de futuros supone un reto provocador dado que CENTRO cuenta con 13 años de experiencia en la formación de profesionales del diseño, la comunicación y la mercadotecnia; como parte de su proceso de maduración, la institución ha dedicido comprometerse con el diseño en un sentido más abstracto y denso con el propósito de formar a especialistas capaces de utilizar su ingenio (y en esta medida, contribuir a la Economía creativa y a la sociedad del conocimiento) de forma sistemática para contribuir a la generación de realidades sociales más ad hoc a los futuros que consideramos deseables (futuribles).

Palabras clave: prospectiva, diseño de futuros, pensamiento complejo, innovación social, retos del milenio 


\begin{abstract}
We present the experience of the researching and making of the Futures Design specialty, graduate program for the training of experts in solving social problems based on the methods of foresight, set of tools and techniques to systematize the imagination to design possible, probable or preferable futures. We concluded that in itself, the experience aims to provide a solution to a cultural problem that significantly permeates all walks of life: shortterm vision. The presentation covers the background of the project, the description of the research process, program exposure and the current status of the project, which takes place in the institutional framework of CENTRO, an institution dedicated to the professionalization of the creative economy.

Among the most important results of this experience that can be identified, in addition to the program itself, which is already current and duly validated by the Ministry of Public Education in Mexico, the formation of a cloister of national and international teachers highly motivated and committed to the experience; the integration of an advisory board with representatives of the most important think-tanks on futurology around the world (the World Future Society, the World Future Studies Federation, Institute for the Future, among others) and incorporating CENTRO to the international discussion in the matter of futures, understanding the future as objects of complex design despite its non-existence, are integrated-conjured- to material reality through narrative, objects, prototypes, scenarios and other material expressions that help guide decision-making in the present .

The designing of futures poses a provocative challenge since CENTRO has 13 years of experience training professionals in design, communication and marketing; as part of their maturation process, the institution has decided to commit to design in a more abstract and dense sense in order to train specialists capable of using their wits (and to this extent, contribute to creative economy and society knowledge) systematically to contribute to the creation of ad hoc social realities for a future we consider desirable (futuribles).
\end{abstract}

Keywords: prospective, futures design, complex thinking, social innovation, millennium challenges

\title{
1. Antecedentes
}

Centro de Diseño, Cine y Televisión (CENTRO) es una institución educativa privada, fundada en el 2013 en la Ciudad de México con la misión de formar a profesionales de la Economía creativa, esto es, personas capaces de participar activamente en cadenas productivas que encuentran en el ingenio su principal materia prima (Howkings, 2016). En este vibrante ámbito se incluyen los diseños, la comunicación audiovisual, la industria editorial, la industria de los videojuegos, el desarrollo de software, entre otras actividades clave.

Dada su naturaleza, la Economía creativa encuentra en la propiedad intelectual e industrial uno de sus indicadores más importantes, de ahí que las universidades (más aun tratándose de instituciones con una 
fuerte vocación hacedora, como es el caso de CENTRO) jueguen un papel fundamental como nichos de la clase super-creativa (Florida, 2014).

Actualmente, CENTRO cuenta con 7 programas de licenciatura y 11 de posgrado, todos relacionados con este sector, capaz de propiciar el desarrollo local y regional. De hecho, México se considera un país altamente competitivo (UNESCO, 2013) en esta materia, si bien aún no alcanza su máximo desarrollo en este ámbito.

En el 2013, el equipo de la coordinación de investigación de CENTRO recibió la encomienda de diseñar un posgrado de avanzada. Para cumplir con esta empresa, el equipo realizó una investigación de la cual se dará cuenta a continuación, cuyo propósito era crear el plan de estudios al cual se referirá más adelante, logrando que la propuesta fuera tan inspiradora como para que los propios profesores que la diseñaron quisieran cursar el posgrado.

\section{La investigación}

El equipo de investigación a cargo de la Especialidad en diseño del mañana de CENTRO estuvo conformado por 2 comunicólogas, 1 mercadólogo, 2 diseñadores, 1 animador y 1 administradora de empresas, todos comprometidos con la causa común de diseñar un programa inspirador, provocador, radical y altamente competitivo.

En principio, el equipo recopiló y revisó minuciosamente 46 programas de posgrado nacionales e internacionales, todos orientados al desarrollo de capacidades creativas aplicadas a los negocios, al diseño de políticas públicas y al diseño de soluciones a problemas complejos, entre otras salidas. De este primer universo se seleccionaron 10 programas especialmente interesantes que en aquel momento se impartían en 9 países (incluído México), ya sea en modalidad de maestría o especialidad. Dichos programas nos permitieron identificar una estructura común que sirvió como esquema inicial para bocetar el plan de estudios.

Por otra parte el equipo analizó la trayectoria profesional de 4 egresados de dichos programas para inspirar el perfil del egresado de CENTRO y realizó entrevistas a expertos acerca de cuál es el ámbito laboral y el potencial del mercado para los especialistas en diseño prospectivo.

La etapa de entrevistas resultó crucial para orientar la investigación, ya que CENTRO cuenta con una vasta experiencia en Economía creativa pero su trayectoria en prospectiva es muy incipiente aún. Por lo tanto nos acercamos a destacadas organizaciones en este ámbito de especialidad:

1. Club de Roma. Organización sin fines de lucro fundada en 1968 por científicos y expolíticos interesados en mejorar el futuro a largo plazo. En la década de los 70 encomendaron el informe Los límites del crecimiento. (Rome, $\mathrm{n} / \mathrm{d}$ )

2. World Future Society. Organización sin fines de lucro fundada en 1966, anualmente publica una serie de informes relativos al futuro remoto y promueve la investigación en esta materia.

3. World Future Studies Federation. Organización sin fines de lucro fundada en 1973, con presencia en 60 países, promueve la generaciónd de futuribles, el intercambio de ideas y la generación de recomendaciones para organismos internacionales en materia de prospectiva. (Federation, n/d)

4. Millenium Project. Think tank internacional fundado en 1996, conformado por 40 nodos de científicos y políticos alrededor del mundo, quienes año con año publican estudios en relación al 
futuro remoto y desarrollan diversos métodos para el diseño de futuribles. Entre otras actividades, diseña, difunde y reflexiona en torno a los retos del Milenio. (Project, n/d)

5. Fundación Javier Barros Sierra. Asociación científica y tecnológica fundada en 1975 que agrupa a expertos dedicados a la prospectiva, específicamente en lo que hace al futuro a largo plazo de México. (Sierra, n/d)

6. Institute for the Future. Think tank sin fines de lucro dedicado a la investigación y la capacitación en materia de estudios del futuro. (Future, n/d)

En los primeros cinco casos, estas organizaciones cuentan con representación en México, de manera que CENTRO se acercó a sus representantes locales con excelente recepción. En el último caso, la fase de investigación coincidió con una gira de Institute for the Future a México, en la cual CENTRO fungió como sede y estableció contacto con el equipo de investigación del instituto.

Esta exploración arrojó resultados muy interesantes que a continuación se refieren.

Los estudios del futuro en México cuentan con una gran tradición que se remonta a la década de los sesenta, durante la cual destaca el trabajo de expertos como Tomás Miklos (Miklos, 1994), Guillermina Bahena (Bahena, 2012), Concepción Olavarrieta (Olavarrieta, 2014)y Antonio Alonso Concheiro (Millán, 2006) (quienes a la fecha se encuentran en activo), sin embargo en las últimas dos décadas el auge de la disciplina ha disminuido de forma significativa, identificándose pocos especialistas jóvenes en la materia. Entre los escasos nombres podemos mencionar el trabajo de Alethia Montero (World Future Society), Margarita Arroyo (Miklos, 2008) y Patricio Buenrostro (Embajadas del futuro), quienes han desarrollado su trabajo al alero de los expertos en principio mencionados y/o en colaboración con las organizaciones referidas.

¿Por qué hay tan pocos futurólogos en México? Nos preguntamos. ¿Dónde se forman los futurólogos existentes? La respuesta fue que en el extranjero o en el único programa de maestría vigente en México (en Monterrey, al norte del país). Sin lugar a dudas, la falta de oferta académica en la materia se hizo notar.

El equipo estaba en busca de inspiración y guía. Encontramos a expertos entusiastas y generosos, convencidos de la necesidad de lanzar un nuevo programa y dispuestos a colaborar su diseño; más tarde, estos expertos configuraron el actual consejo consultivo del posgrado. El análisis de las trayectorias de los propios consejeros sirvió para orientar el desarrollo del plan de estudios y crear el perfil del egresado, concebido como un experto capaz de idear escenarios futuros y asesorar a los sectores público, privado y civil en la implementación de decisiones presentes que hagan posible la consecución de los escenarios más deseables.

\subsection{Acerca de los estudios del futuro}

Por estudios del futuro entenderemos un "tipo de investigacion especial mente creativa, orientada a la exploracion del porvenir, con el objeto de proporcionar informacion relevante, en una perspectiva a muy largo plazo que permita apoyar la toma de decisiones" (Miklos, 1994).

El propósito de estos estudios es brindar información útil para los tomadores de decisiones, considerando los escenarios posibles, probables y preferibles como alternativa. Vale la pena destacar que este campo disciplinario parte de la premisa de que los futuros no existen y en esta medida pueden diseñarse como objetos complejos: ¿qué mejor reto para un profesional de la economía creativa que utilizar su ingenio para pensar en escenarios que no existen, pero podrían existir si trabajamos en su consecución? El 
ejercicio supone el uso del ingenio, pero también de procedimientos formales para fundamentar ese acto creativo.

Con su incursión en el diseño de futuros, CENTRO se propone dar un paso más en su crecimiento en la formación de problem solvers. Por supuesto, esto implica un gran reto, el primer de ellos consiste en instar a los estudiantes para que se alejen un poco de su zona de confort y utilicen su imaginación y su capacidad analítica para pensar en soluciones hasta entonces fuera de su marco de referencia. Con este paso, la propia institución asume el reto de diseñar soluciones para un mundo en el que quizás el diseñador ya no se encontrará en el momento de la implementación: ¿qué tipo de consumo tendrán los nietos de la generación Z? ¿cómo debe ser la indumentaria que necesitan los habitantes de Marte? ¿qué políticas públicas deben implementarse ahora para lograr un manejo sustentable del agua dentro de 50 años? ¿cómo serán las aulas en las universidades dentro de dos generaciones? Son algunas preguntas que nuestros estudiantes deben plantearse para idear soluciones.

\section{El programa}

\subsection{Objetivos}

Los objetivos del programa se enuncian así: El alumno diseñará escenarios probables, posibles o deseables en torno a una problemática social relevante y concreta; en un marco organizacional determinado; el alumno conocerá, practicará y evaluará procedimientos de análisis efectivos para el diseño prospectivo y la innovación.

En lo que hace a los objetivos emocionales, nos propusimos que los alumnos se sintieran altamente motivados, desconcertados, ansiosos, estimulados y comprometidos con la experiencia, pensada para grupos de máximo 10 personas, lo cual permite hacer adaptaciones para garantizar su carácter personalizado.

\subsection{Perfil de egreso}

El egresado será capaz de construir escenarios novedosos, creativos, originales aplicando diversas herramientas e instrumentos del diseño prospectivo, la invención y la innovación para la solución de problemas sociales específicos, usando como base las tendencias y certezas estructurales existentes.

Podrán ser consultores privados, asesores de los tres niveles de gobierno, guionistas de televisión, radio y cine para la elaboración de escenarios en la incertidumbre y de eventos inesperados. Los egresados de esta especialidad deben ser capaces de pensar en un objeto que no existe: el futuro. O para ser más precisos, los futuros (posibles o deseables), considerando que es una entidad que no existe, pero que puede diseñarse para fundamentar la toma de decisiones en el presente. 


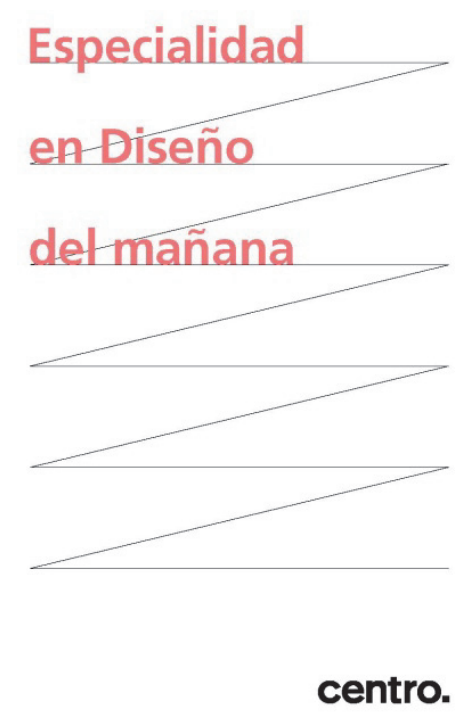

Fig. 1. Pieza promocional

\subsection{Currícula}

El programa de la especialidad tiene dos centros: la formación metodológica y la práctica de los métodos. En ambos casos se disenarón materias robustas alrededor de las cuales se concibieron asignaturas complementarias.

El primer boceto del programa fue diseñado por el equipo de investigación de CENTRO con base en los resultados del estado del arte, posteriormente fue comentado por el Institute for the Future, la World Future Society y la World Future Studies Federation. Dado que estas organizaciones no necesariamente coinciden en puntos de vista, los diferentes comentarios contribuyeron a lograr una propuesta diversa y balanceada en contenidos.

\begin{tabular}{|c|c|}
\hline Segmento & Contenido \\
\hline \multirow[t]{5}{*}{ Propedéutico } & Pensamiento de diseño \\
\hline & Análisis de textos \\
\hline & Aparato crítico \\
\hline & Retos del milenio \\
\hline & Introducción a la prospectiva \\
\hline \multirow[t]{6}{*}{ Segundo semestre } & Historia de los estudios de futuros \\
\hline & Antropología simbólica \\
\hline & Pensamiento sistémico y teorías del cambio \\
\hline & Métodos prospectivos \\
\hline & Inteligencia prospectiva en la incertidumbre y la complejidad \\
\hline & Construcción de escenarios \\
\hline
\end{tabular}




\begin{tabular}{|l|l|}
\hline Primer semestre & Contexto geopolítico \\
\cline { 2 - 2 } & Pensamiento anticipatorio e innovación \\
\hline & Procesos innovadores de planeación prospectiva estratégica \\
\hline & Laboratorio de innovación social \\
\hline & Escenarios de innovación disruptiva \\
\hline & Narrativa y representación de escenarios a largo plazo \\
\hline
\end{tabular}

Fig.2. Estructura del plan de estudios. Fuente: Elaboración propia

El programa se imparte en 192 horas presenciales (sin considerar clases magistrales, conferencias, talleres y otras actividades complementarias) organizadas en dos sesiones semanales, durante dos semestres.

Cabe hacer notar que las personas que cursan este programa deben generar una carpeta de proyecto para graduarse y que dicho proyecto debe centrarse en una solución con alcance social. Dicha carpeta debe contener todos los entregables generados en cada uno de los cursos: futuribles narrados, futuribles representados visualmente, prototipos (dibujados, impresos en 3D u otros soportes según el caso), informes de investigación, estudios de caso, entre otros posibles entregables del diseño de futuros.

A lo largo del programa, también se insta a los alumnos para que incursionen en experiencias de diseño que de alguna manera los vinculen con la red de especialistas en prospectiva, tales como rondas Delphi, talleres para el diseño de futuribles, consultorías guiadas por sus profesores, entre otras experiencias de formación. Ello les permite familiarizarse con la visión de los expertos y sensibilizarse frente a las problemáticas que los futurólogos suelen atender.

\subsection{Claustro de profesores y grupo de alumnos}

El equipo de profesores a cargo del programa es diverso. Todos los docentes son posgraduados con nivel mínimo de Maestría y el $40 \%$ de la planta cuenta con formación específica en Prospectiva. Contamos con psicólogos, historiadores, filólogos, filósofos, diseñadores, antropólogos, internacionalistas y sociólogos en el equipo.

Vale la pena mencionar que además del equipo básico, todos los alumnos cuentan con un mentor nacional y uno internacional para el seguimiento de sus proyectos.

Por su parte, los alumnos de la primera generación son diseñadores, administradores y comunicadores.

\subsection{Actividades realizadas hasta el momento}

Al cierre de este texto, la primera generación de la especialidad concluye su primer semestre. Los alumnos están bocetando sus propuestas, mismas que serán discutidas con sus mentores en las próximas semanas.

Hasta el momento hemos realizado una conferencia magistral y tenemos programas dos más con expertos del sector. Adicionalmente los alumnos cursarán dos talleres para la práctica de técnicas prospectivas específicas y complementarán su formación con una clase magistral más.

En las próximas semanas también se llevará a cabo una mesa redonda con presentación de libros, uno de los cuales presenta el cruce perfecto entre la prospectiva y el diseño conceptual: se trata del Códice de Eduardo Terrazas (Terrazas, 1975), obra gráfica inspirada en el clásico Los límites del crecimiento (Behrens, 1972). 
En el segundo semestre, los alumnos entrarán al laboratorio de innovación, en el cual prototiparán y evaluarán sus escenarios. Asimismo tendrán dos conferencias magistrales y un taller más antes de estar en condiciones de presentar sus carpetas finales.

En paralelo, hemos iniciado el seminario de profesores de la especialidad, durante el cual intercambiamos contenidos de interés, profesionalizamos a la planta docente en técnicas prospectivas y en general, facilitamos el trabajo colaborativo de cada miembro del staff para integrar productos significativos y congruentes con el programa.

El camino ha sido arduo e intenso, sobre todo si pensamos que la generación está conformada por seis estudiantes. Sin embargo, ya tenemos lista de espera para la siguiente generación y esta primera experiencia nos ha permitido perfeccionar el modelo educativo y por ende los contenidos y las experiencias de formación.

Lo decisivo será, bien lo sabemos, los entregables que produzcan, cuya calidad será evaluada por el consejo consultivo de la especialidad.

\section{Conclusión: tan difícil como diseñar lo intangile}

Comenzaré por aclarar que la denominación "diseño del mañana" es imprecisa y se estableció con fines comerciales, lo correcto sería referir al diseño de futuros. El mañana es muy inmediato, la prospectiva promueve una idea más remota de los futuros; por otra parte, el mañana es uno y los futuros posibles, probables o preferibles son múltiples.

Hecha esa precisión, se concluye que un especialista en prospectiva crea objetos complejos (lo mismo una taza que una situación o un sistema) y para ello combina su imaginación con técnicas formales para fundamentar la propuesta. Es un diseñador en toda forma que genera información útil para otras personas, incluso para sí mismo, pero que enfrenta el reto de crear algo para lo que quizás no existen referencias (materiales o tecnologías que no han sido inventadas, por ejemplo).

Nuestra mayor área de oportunidad como coordinadores del programa es resistir a la indiferencia del mercado sobre la necesidad inminente de diseñar los futuros, proclive como es la cultura mexicana al pensamiento a corto plazo. Asimismo, la profesionalización del equipo de CENTRO para aplicar las técnicas prospectivas para el desarrollo de la propia institución es aún una tarea por completarse, si bien estamos trabajando en ella.

En mi opinión, el solo hecho de que CENTRO haya tomado el riesgo de emprender este proyecto, no inédito pero sí radical en relación a la inercia de la cultura mexicana, resulta esperanzador. ¿Cómo enfrentamos esta gran responsabilidad? Con mucha imaginación, visión de largo plazo y pensamiento sistemático, como los futurólogos. 


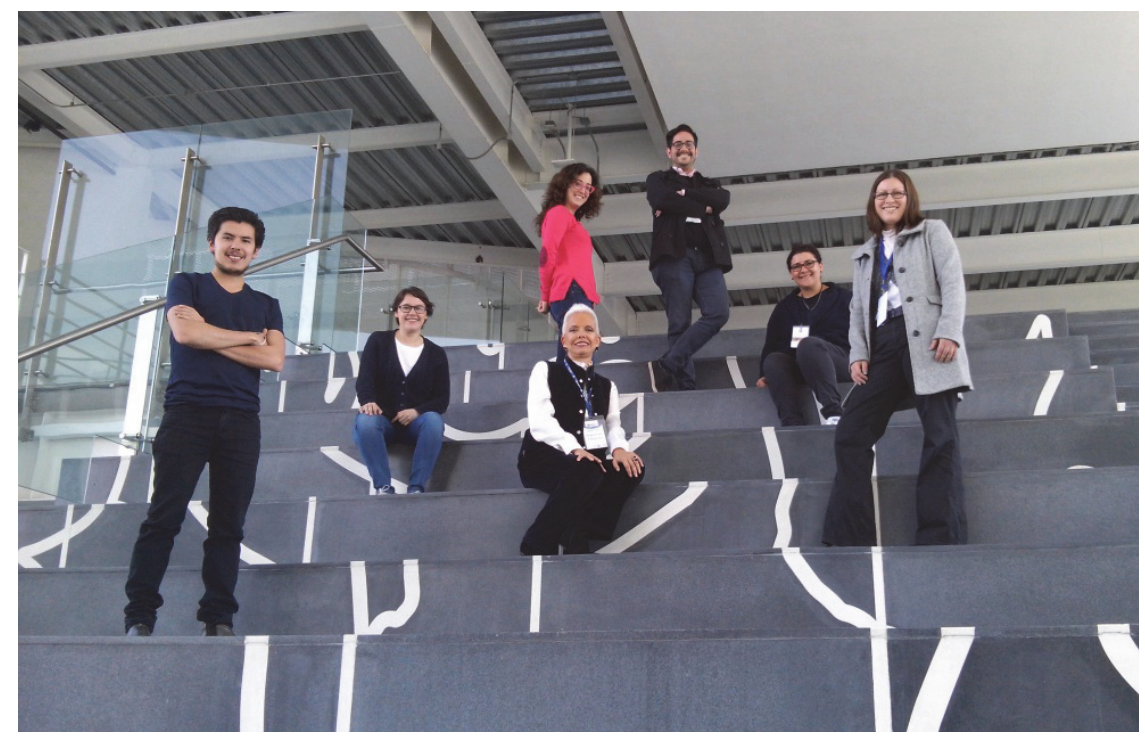

Fig.3. Estudiantes con la futuróloga Concepción Olavarrieta. Fuente: CENTRO

\section{Referencias}

BAHENA, G., (2012). Inteligencia prospectiva. 1a ed. México: EAE.

BEHRENS, W. R. J. M. D. (1972). The Limits of Growth. 1a ed. n/a: Potomac Associates.

Federation, W. F. S., n/d. World Future Studies Federation. [Online] Available at: http://www.wfsf.org/ [Accessed 28 abril 2016].

FLORIDA, R., (2014). The Rise of the Creative Class-Revisited. Nueva York: Basic Books.

FUTURE, I. F. T., n/d. Institute for the Future. [Online] Available at: http://www.iftf.org/home/[Accessed 28 abril 2016].

HOWKINGS, J., (2016). The Creative Economy: How People Make Money from Ideas. Londres: Penguin.

MIKLOS, T. Y. A. M., (2008). Madrid toma la plaza. [Online] Available at: http://madrid.tomalaplaza.net/files/2011/07/WORKING PAPERS 8.pdf[Accessed 2804 2016].

MIKLOS, T. Y. T. M., (1994) Planeación prospectiva, una propuesta para el diseño del futuro. 1a ed. México: Limusa.

MILLÁN, K. Y. C. A. (2006). México 2030. Nuevo siglo, nuevo país. 1a ed. México: FCE.

OLAVARRIETA, C. G. J., (2014). Futuros. Diccionario enciclopédico mundial sobre prospectiva. 1a ed. Washington: Millenium Project.

PROJECT, M., n/d. Millenium Project. [Online] Available at: http://www.unmillenniumproject.org/[Accessed 28 abril 2016].

ROME, C. O., n/d. Club of Rome. [Online] Available at: http://www.clubofrome.org/[Accessed 28 abril 2016].

SIERRA, F. J. B., n/d. Fundación Javier Barros sierra. [Online] Available at: http://www.fundacionbarrossierra.org.mx/ [Accessed 28 abril 2016].

SOCIETY, W. F., n/d. World Future Society. [Online] Available at: http://www.clubofrome.org/[Accessed 28 abril 2016]. 
La prospectiva como diseño de lo intangible. El caso Centro.

TERRAZAS, E., (1975). Códice para la solidaridad y el desarrollo. 1a ed. México: Ediciones frontera.

UNESCO, (2013). Creative Economy Report. Nueva York: UNESCO. 